hep-ph/9707542

McGill-97/13

\title{
Neutrino Propagation Through Helioseismic Waves
}

\author{
P. Bamert, C.P. Burgess and D. Michaud \\ Physics Department, McGill University \\ 3600 University St., Montréal, Québec, Canada, HзA 2 T8.
}

\begin{abstract}
Motivated by earlier calculations showing large effects when neutrinos propagate through fluctuating media, we perform here a detailed analysis of how density fluctuations in the sun in the form of helioseismic waves can modify the MSW solution to the solar neutrino problem. We find negligible effects for the MSW spectrum, even under extreme circumstances. There are two main reasons why our conclusions differ from earlier analyses. First, most helioseismic waves do not affect neutrino propagation because their amplitude is too small in the MSW resonance region, which is the only region to whose fluctuations neutrinos are sensitive. There is one class of waves which may be subject to an instability, however, and so can have significantly larger amplitudes. But the wavelength for these waves is so long that it invalidates the previous methods of calculation. Our more complete calculation significantly reduces the prediction for their influence on neutrino propagation.
\end{abstract}




\section{Introduction and Summary}

Taken together, the combined information from the four pioneering solar neutrino experiments [1] suggests a neutrino spectrum which deviates strongly from astrophysical predictions [2]. Purely astrophysical attempts to reconcile the data with theory fail because of the virtually complete suppression of ${ }^{7} \mathrm{Be}$ neutrinos seen by the experiments together with the observed nonzero ${ }^{8} \mathrm{~B}$ neutrino flux. These two observations are hard to reconcile with one another using a solar model since the observed ${ }^{8} \mathrm{~B}$ neutrino flux is made from the same chain of nuclear reactions which would have also produced the missing ${ }^{7} \mathrm{Be}$.

Particle physics explanations of the neutrino spectrum don't suffer from the same difficulty. The most successful of these explanations is the resonant conversion of neutrino flavours within the solar medium, the so called MSW mechanism [3]. It provides an excellent description of the experiments using theoretically plausible neutrino parameters.

The original MSW analysis starts from a mean-field treatment of the background through which the neutrinos propagate. More recent work has since investigated how corrections to the mean-field picture might influence the neutrino survival probability [4], [5], [6], [7], [8]. For technical reasons these studies focus on fluctuations about the mean which have small spatial correlation lengths compared to the distances over which neutrinos appreciably oscillate. Within this approximation the conclusions obtained were that fluctuations of this type in the solar electron density can indeed significantly modify the MSW neutrino spectrum, provided their amplitude near the MSW resonance point can be as large as a few percent of the background density.

The missing element to these studies has been the identification of a realistic source of such fluctuations within the sun. Unfortunately, two things work against having fluctuations affect neutrino propagation in the sun. First, ordinary thermal fluctuations give negligibly small contributions [8] because of the extremely small interaction cross section between neutrinos and other particles at solar densities. Second, in order to appreciably disturb neutrino oscillations, macroscopic fluctuations would have to occur in the same parts of the sun as does the MSW resonance itself [8]. Unfortunately, most of the fluctuation sources which have been proposed are associated with the enormous turbulence of the sun's outlying convective layer [9], and so are too far from the MSW resonance regions to have much effect. More exotic proposals which involve mixing and convection in the solar core, have recently been ruled out by helioseismic data [10].

To our knowledge, there is only one source of fluctuations which is known to be present in our sun, and which is not known to be excluded from the solar interior: helioseismic waves [11]. Furthermore, estimates based on the short-correlation-length analysis indicate 
[6], [8] that it would suffice for such waves to have amplitudes of a few percent in order for them to alter solar-neutrino propagation.

The present paper provides a more careful calculation of solar-neutrino propagation through realistic helioseismic waves, to accurately determine the potential size of their influence on MSW oscillations. We explicitly compute seismic waves profiles within the sun in order to reliably correlate their amplitude at the resonance point with observations (which are made at the solar surface). We then numerically evolve neutrinos through these profiles to identify their effect on MSW oscillations. Since it turns out that most of the waves of interest have wavelengths much longer than typical neutrino oscillation scales, we perform the calculation without making use of the short-correlation-length approximation.

Our results, in a nutshell, are as follows:

- Standard MSW neutrino oscillations are not affected by helioseismic waves, ${ }^{1}$ for the following reasons.

- The observed helioseismic waves ( $p$-waves) decrease in amplitude as one moves into the sun, with the result that their observed size at the solar surface makes them too small to produce observable effects in the resonance region.

- An important class of waves ( $g$-waves) grows in amplitude with increasing solar depth. Furthermore, some of these may be subject to an instability which makes their amplitude orders of magnitude larger than their stable compatriots. The wavelengths of these waves are typically too large to permit working in the small-correlation-length limit. However, it turns out that small-correlation-length calculations, when applied beyond their domain of applicability, give an overestimate of neutrino-propagation effects, and so a full calculation gives negligible effects for neutrinos, even from these strongly-excited modes.

- Furthermore we find that a density perturbation most strongly affects neutrino propagation when its correlation length is of the order of the length scale at which the shortcorrelation-length approximation fails, typically within an order of magnitude of the neutrino oscillation length.

The outline of the rest of this paper is as follows. In $\S 2$ we recap how density fluctuations can influence neutrino propagation, including a comparison between 'exact' results and those obtained using the formalism adapted to short correlation lengths (called henceforth the master-equation approach). This comparison shows precisely how long correlation lengths must be to invalidate the master-equation approach, as well as how bad its pre-

1 One way small effects can accrue incrementally to appreciably influence neutrino oscillation is if they act repeatedly, such as for a parametric resonance (1). This kind of mechanism appears to play no role when neutrinos pass through helioseismic waves having realistic amplitudes, however. 
dictions become when applied beyond its domain of applicability. $§ 3$ gives a whirlwind summary of what is known about the internal structure of the sun, and the seismic waves it supports. $\S 4$ describes the numerical aspects of our simulations of neutrino propagation through helioseismic waves. The resulting conclusions are then drawn in $\S 5$.

\section{Neutrino Propagation in the Presence of Density Fluctuations}

This section gives a short review of the influence of density fluctuations on solar neutrino propagation. We treat separately the case where the correlation length is short, and describe the master-equation formalism which applies in this limit. The results in this limit are then compared with a more complete calculation for a simple model of solar fluctuations.

\section{1) Solar Neutrinos and Fluctuations}

Conceptually, there are two distinct types of fluctuations to consider when any particle propagates through a medium like the sun. First, if the particle frequently interacts with the medium's constituents, and these constituents are randomly distributed, then the effects on a specific particle of multiple interactions with the medium may be described by a statistical average over a fictitious ensemble of equivalent media. An example of this sort is furnished by photons propagating through the sun, since each photon scatters many times while escaping from the solar interior. Although this kind of multiple scattering is believed to happen to neutrinos moving through the interior of a supernova, it does not apply to neutrinos in the sun.

Alternatively, if the mean properties of the medium vary in space or time, the mean features as seen by successive particles can vary. The average response of a detector to many such particles may also be described in terms of an average over the ensemble of 'media' seen by the individual particles. This is the kind of average which is relevant for solar neutrino propagation through helioseismic waves. Any one neutrino interacts extremely rarely, and escapes the sun in a matter of seconds. Helioseismic waves, on the other hand, alter the electron density over timescales of minutes or hours, and vary in space over distances comparable with the solar radius.

Since any one neutrino interacts so weakly with the solar environment as to see negligible fluctuations, the time evolution of its density matrix is described by the usual Schrödinger equation:

$$
\rho(t, n)=U(t, n) \rho(0) U^{\dagger}(t, n),
$$


where $\rho$ is the neutrino density matrix, $t$ denotes time, $U(t, n)$ is a unitary evolution operator, and $n$ generically denotes all of those features of the solar medium on which the evolution can depend parametrically, and which can change in the time between the transit of different neutrinos. For example, for neutrino oscillation experiments $\rho$ may be taken to be a matrix in neutrino-flavour space, and $U$ is the solution to the evolution equation

$$
\frac{\partial U}{\partial t}=-i V(t, n) U
$$

where $V(t, n)=V_{V A C}+V_{M S W}(t, n)$, with $V_{V A C} \approx k+\frac{m^{\dagger} m}{2 k}+\ldots$ giving the vacuum evolution matrix for an ultrarelativistic neutrino of three-momentum $k$ and mass matrix $m$. Similarly, $V_{M S W}(t, n) \equiv \sqrt{2} G_{F} g^{e}\left\langle n_{e}(t)\right\rangle$ is the leading-order effective interaction with the mean matter background, and $g^{e}=\operatorname{diag}(1,0)$ is a matrix describing the charged-current coupling to the electron density $n_{e}(t)$. For simplicity we consider here only the case of two active neutrino species.

The final response of a neutrino detector is then obtained by averaging the appropriate observable over an ensemble of values for the variables, $n$, weighted by a probability distribution, $p(n)$ :

$$
\langle\mathcal{O}\rangle(t)=\int d n p(n) \operatorname{Tr}[\mathcal{O} \rho(t, n)]=\operatorname{Tr}[\mathcal{O}\langle\rho(t)\rangle]
$$

Much of the physics of the resulting fluctuations is encoded in the probability distribution, $p(n)$. The most commonly-used choice [4], [6], [7], [8] is to assume density fluctuations which are uncorrelated in space. For the present applications we instead follow Ref. [8] and expand the electron density in terms of a complete basis of helioseismic modes:

$$
n_{e}(t)=\left\langle n_{e}(t)\right\rangle\left[1+\sum_{j} \mathcal{C}_{j} \phi_{j}(t)\right]
$$

where the coefficients $\mathcal{C}_{j}$, are assumed to be uncorrelated random variables which are Gaussian distributed, with vanishing mean: i.e. $\left\langle\mathcal{C}_{j}\right\rangle=0$ and $\left\langle\mathcal{C}_{j} \mathcal{C}_{k}\right\rangle=\mathcal{D}_{j} \delta_{j k}$. 33 is devoted to the explicit construction of the basis functions which are appropriate for helioseismic waves.

In subsequent sections we directly solve these equations for $U(t, n)$ and $\rho(t, n)$ to find the electron-neutrino survival probability after transit through the sun. Before doing so, we 
next pause to describe the master-equation approach, which has been the main theoretical tool used in previous investigations.

\section{2) Short Correlation Lengths: The Master Equation}

We summarize here the formalism of [4], [6], [7] and [8], in which references the interested reader may find more details.

The master-equation approach arises most naturally for physical systems where the ensemble average is meant to describe a transient particle's multiple interactions with the ambient material. For this kind of system it is natural to formulate a a coarse-grained time derivative, $D \rho / D t$, of the density matrix which incorporates the average over shorttimescale fluctuations. The result turns out to give a reasonable description of solar neutrino propagation through short-range density variations, even though neutrinos do not multiply interact with the solar medium.

The starting point is the observation that treating the local electron density as a random variable introduces a correlation length into any ensemble averages. More precisely, suppose that the correlation $\left\langle\delta V(t) \delta V\left(t^{\prime}\right)\right\rangle$ (where $\delta V=V-\langle V\rangle$ ) becomes negligible whenever $\left|t-t^{\prime}\right|$ is greater than some characteristic scale, $\tau_{c}$. When coarse-grained over times longer than $\tau_{c}$, the derivative, $D \rho / D t$, evaluated at time $t$, depends only on the value of $\rho(t)$, and has no memory of how $\rho$ has evolved over earlier times. Furthermore, when $\tau_{c}$ is sufficiently short compared to the time scales in the interactions, the coarse-grained derivative, $D \rho / D t$, may also be evaluated perturbatively in $V$.

As applied to the neutrino density matrix in two-by-two flavour-space, this leads to the master equation [4], [6], [7], [8]:

$$
\frac{D \rho}{D t}=-i\left[V_{V A C}+V_{M S W}(t), \rho\right]-2 G_{F}^{2} \mathcal{A}(t)\left[\left(g^{e}\right)^{2} \rho+\rho\left(g^{e}\right)^{2}-2 g^{e} \rho g^{e}\right]+\mathcal{O}\left(V^{3}\right)
$$

The first term of eq. (5) describes the usual MSW evolution. The coefficient $\mathcal{A}(t)$ of the second term is the correlation integral

$$
\mathcal{A}(t) \equiv \int_{t^{\prime}}^{t} d \tau\left\langle\delta n_{e}(t) \delta n_{e}(\tau)\right\rangle
$$

which represents the fluctuation effects.

Eq. (5) can be directly integrated if the following two approximations hold: $(i)$ the neutrino evolution away from MSW resonances can be treated in the adiabatic approximation, and ( $i i)$ the resonance region is sufficiently narrow as to justify approximating the 
electron density profile there as a linear function of distance along the neutrino path. In this limit one obtains a prediction for the probability that an electron-neutrino produced at time $t^{\prime}$, survives to time $t$, which generalizes the well-known Parke formula [13] to include the effects of fluctuations [8]:

$$
P_{e}\left(t, t^{\prime}\right)=\frac{1}{2}+\left(\frac{1}{2}-P_{J}\right) \lambda \cos 2 \theta_{m}\left(t^{\prime}\right) \cos 2 \theta_{m}(t)
$$

Here $P_{J}=\exp \left[-\frac{\pi}{2}\left(\frac{\sin ^{2} 2 \theta_{V}}{\cos 2 \theta_{V}}\right)\left(\frac{\delta m^{2} h}{2 k}\right)\right]$ is the jump probability, where $h$ is the scale height for the averaged electron density, and $\delta m^{2}$ is the squared-mass difference between the two neutrino mass eigenstates in vacuum. $\theta_{V}$ is the vacuum mixing angle, while $\theta_{m}(t)$ is the matter mixing angle evaluated at the position occupied by the neutrino at time $t$. The coefficient, $\lambda$, equals one in the absence of fluctuations, but more generally is given by

$$
\lambda \equiv \exp \left[-2 G_{F}^{2} \int_{t^{\prime}}^{t} d \tau \mathcal{A}(\tau) \sin ^{2} 2 \theta_{m}(\tau)\right]
$$

Notice that eq. (8) implies that fluctuation effects are strongest at resonance where $\sin ^{2} 2 \theta_{m}$ is maximised. We find in subsequent sections that this conclusion still holds when we go beyond the limitations of this perturbative approach.

\section{3) When the Master-Equation Technique Fails}

Eq. (5), and so also eqs. (7) and (8), fail once the correlation time, $\tau_{c}$, becomes too large. A simple way to see that this must be so is to notice that $\mathcal{A}(t)$ need not, in general, be positive, and so for large correlation lengths — and so for large $\mathcal{A}(t)-\lambda$ can be greater than one. This, in turn, can make $P_{e}$ lie outside the interval $[0,1]$, and so be unphysical. This section aims to determine more precisely when, and by how much, predictions based on the master equation, eq. (5), fail once correlation lengths become large.

A first estimate for when these formulae fail may be obtained by figuring the size of the $O\left(V^{3}\right)$ term in $D \rho / D t$. For the purpose of so doing, we consider a simple model of fluctuations in which the solar medium is divided into regions ('cells') of linear size $\ell$. We imagine an ensemble of density fluctuations in these cells, which are uncorrelated from cell to cell. Quantitatively, we take

$$
\left\langle\delta n_{e}(\mathbf{r}) \delta n_{e}\left(\mathbf{r}^{\prime}\right)\right\rangle=\left\{\begin{array}{cc}
\epsilon^{2}\left\langle n_{e}(\mathbf{r})\right\rangle\left\langle n_{e}\left(\mathbf{r}^{\prime}\right)\right\rangle & \text { if } \mathbf{r} \text { and } \mathbf{r}^{\prime} \text { lie in the same cell; } \\
0 & \text { otherwise }
\end{array}\right.
$$


With this choice, the fluctuation term in eq. (5) is of order $G_{F}^{2} \mathcal{A}(t) \sim \ell\left(\epsilon G_{F}\left\langle n_{e}\right\rangle\right)^{2}$, while the neglected $O\left(V^{3}\right)$ terms are $\sim \ell^{2}\left(\epsilon G_{F}\left\langle n_{e}\right\rangle\right)^{3}$.

This very crude estimate therefore indicates that eq. (5) fails for fluctuations satisfying $\ell \gtrsim 1 /\left(\epsilon G_{F}\left\langle n_{e}\right\rangle\right)$. This estimate agrees passably well with the more detailed comparison between the master-equation method, and an 'exact' ensemble average, which we now present.

To obtain a feel for the numbers, taking $1 /\left(G_{F}\left\langle n_{e}\right\rangle\right) \sim 300 \mathrm{~km}$, which is typical at resonance, and $\epsilon \sim 10 \%$ this condition becomes $\ell>3,000 \mathrm{~km}$. Helioseismic waves, especially those with the largest amplitudes, have wavelengths (i.e. correlation lengths) at resonance which can be of order $R_{\odot} / 10 \sim 70,000 \mathrm{~km}$, and so easily exceed the size of $\ell$.

We have performed a more quantitative comparison between neutrino-survival predictions using the master equation, or a direct numerical ensemble average performed without approximation over 200 random density profiles of the Cell type. We choose a geometry, in which the neutrino moves along the $z$ axis through a grid of rectangular cells of length $\ell$. We take the mean density profile to fall exponentially with $z$, with a scale height typical of solar models: $h \sim 6.6 \times 10^{4} \mathrm{~km} \sim R_{\odot} / 10$. The fluctuation size is taken as $\epsilon=0.1$.

Figure (1) summarizes the results of this comparison. The horizontal dotted line in this figure gives the standard MSW survival probability in the absence of fluctuations, using the oscillation parameters $\delta m^{2} / 2 k \sim 10^{-6} \mathrm{eV}^{2} / \mathrm{MeV}$ and $\sin ^{2} 2 \theta_{V}=0.01$. The thin solid line expresses the prediction of the generalized Parke formula, eqs. (7) and (8). Notice how the generalized Parke expression approaches the MSW result for small $\ell$, and goes to a fixed value, $P_{e}=0.5$, for large $\ell$.

For comparison, the remaining three curves give the results of a direct numerical average over an ensemble of electron densities, without using the master-equation formalism. For these curves the survival probability for each neutrino is computed using Parke's formula - in the form originally proposed by Parke, without fluctuations — and this result is then numerically averaged over 200 elements of the density ensemble. We have checked our use of the ordinary Parke formula for the survival probability of any one neutrino as it moves through a given density profile by comparing it with the direct numerical integration of the neutrino evolution equations. We find good agreement, although for large-amplitude helioseismic waves this agreement can require the use of Parke's formula for the passage through several MSW resonances.

The three curves obtained in this way differ in how they treat the case where the MSW resonance falls at the boundary of a cell, where the density profile is varying artificially strongly. In the thick solid line, the cells are adjusted to ensure that the MSW resonance 
always falls well away from any cell boundary. By contrast, the dashed line arranges the resonance to always fall at the cell boundary. The dot-dashed line corresponds to randomly positioned cells, whose boundaries may or may not fall at the MSW resonance. Notice these three curves agree with each other except for the largest $\ell$, where the larger effect is obtained when the resonance hits a cell wall. This cell-boundary effect is less pronounced for smoother density profiles. In the absence of cell wall/resonance coincidences (thick solid curve) the ensemble-averaged survival probability approaches the MSW prediction for sufficiently large $\ell$.

The generalized Parke prediction differs most dramatically from the direct ensemble average for $\ell \gtrsim 5,000 \mathrm{~km}$, as may be seen by comparing the thick and thin solid lines in Fig. (1). For larger $\ell$ the numerical simulation agrees with the MSW prediction (in the absence of cell-boundary effects); a feature which is missed by the generalized Parke result (thin solid line). Notice that $5,000 \mathrm{~km}$ agrees reasonably well with the estimate, $\ell \gtrsim 1 /\left(\epsilon G_{F}\left\langle n_{e}\right\rangle\right) \sim 3,000 \mathrm{~km}$, given above, for the scale at which correlations should fail to be well described by master-equation methods.

The lessons from this section are these:

- Master equation methods describe solar neutrinos surprisingly well for sufficiently small correlation lengths, but can dramatically overestimate the deviation from the MSW prediction when the correlation lengths are large.

- Numerically, the dividing line between large and small correlation lengths is of order $3,000 \mathrm{~km}$, which is quite small compared to the length scales over which helioseismic waves can vary.

\section{Helioseismic Waves at a Glance}

Our sun is not as quiet as it seems. On the contrary, it displays a wide variety of nonequilibrium phenomena, many of which might give rise to density perturbations [14]. Unfortunately, most of these solar disturbances are restricted to the solar surface, or to the convective zone (i.e. $r / R_{\odot} \gtrsim 0.7$ ). By contrast, Figure (2) shows the depths of the MSW resonance regions as a function of neutrino energy and mixing parameters, illustrating that all resonances occur well within half a solar radius. It follows that most solar density fluctuations are much too close to the solar surface to play any role in neutrino oscillations. The notable exception to this are helioseismic waves, the enumeration of whose properties is the main subject of this section.

\section{1) Qualitative Discussion}


We next present the main features of helioseismic waves, with our treatment following the excellent review found in Ref. [11].

We start with a brief reminder of the solar structure. The sun can be divided into four main regions. The energy-producing core is at the centre, extending out to roughly 0.2 solar radii. Next comes the radiative zone, for $0.2 \lesssim r / R_{\odot} \lesssim 0,7$, in which the dominant means of energy transfer is radiative. The convective zone follows, for $r / R_{\odot} \gtrsim 0.7$, where convection is the dominant method of energy transfer. Finally, the solar surface occupies a comparatively thin layer near $r / R_{\odot} \sim 1$. The enumeration of these regions is important because the properties of a helioseismic wave are largely governed by the zone in which it sits.

Solar oscillations can be classified into two qualitatively different types of waves, known as ' $p$-' and ' $g$-waves' respectively, depending on the nature of the restoring force which is responsible for the oscillatory behaviour. For $p$-waves pressure is the relevant restoring force, while for $g$-waves it is buoyancy which plays this role. Because of this difference in restoring force for each, $p$ - and $g$-waves tend to be found in different regions of the sun. Since convection is related to an instability in the buoyancy force, $g$-waves are damped within the convective zone, and so are confined to the interior of the sun. $p$-waves, on the other hand, can exist in all four zones, although because the speed of sound increases with depth, only radially-directed waves tend to penetrate deep into the central regions. Both have similar frequencies, with $p$-waves having periods typically below 30 minutes, and $g$-waves with periods longer than this value.

\section{2) Quantitative Features}

Modelling the properties of these waves requires solving the four hydrodynamic equations - expressing the conservation laws of particle number, energy and momentum which govern their properties. The approximate spherical symmetry of the sun allows the solutions to be expressed in terms of spherical harmonics so long as only small perturbations about the static background are considered. The density perturbation, $\delta \rho$, representing a wave can thus be written

$$
\delta \rho(r, \theta, \phi, t)=\sqrt{4 \pi} \varrho_{n \ell}(r) Y_{\ell m}(\theta, \phi) \exp \left(-i \omega_{n \ell} t\right)
$$

where the spherical harmonics $Y_{\ell m}$ are normalized to satisfy $\int d \phi d \theta \sin \theta\left|Y_{\ell m}\right|^{2}=1$. Spherical symmetry ensures that the wave frequency, $\nu=\omega /(2 \pi)$, depends only on the radial and angular quantum numbers, $n$ and $\ell$, but not the azimuthal quantum number, 
$m$. This degeneracy of modes is broken by the rotation of the sun, although this effect is too small to be of further relevance here.

Our simulations are performed using the Cowling approximation, in which the gravitational backreaction of the wave onto itself is neglected. In this approximation, the hydrodynamical equations simplify considerably when they are expressed in terms of the following variable: $\xi=\frac{\left|S_{\ell}^{2} / \omega^{2}-1\right|}{\rho_{0} c^{2} r^{2}} \xi_{r}$, with $\xi_{r}$ denoting the radial displacement of the wave, $\rho_{0}$ representing the unperturbed mass density and $c$ denoting the speed of sound of the background solar medium. The equations can then be written [11]:

$$
\xi^{\prime \prime}(r)+K(r) \xi=0, \text { with } K(r) \approx \frac{\omega^{2}}{c^{2}(r)}\left(\frac{N^{2}(r)}{\omega^{2}}-1\right)\left(\frac{S_{\ell}^{2}(r)+\omega_{c}^{2}(r)}{\omega^{2}}-1\right) .
$$

The buoyancy frequency, $N(r)$, and acoustic frequency, $S_{\ell}(r)$, vary with depth within the sun, and are given explicitly by the following expressions:

$$
N^{2}=\frac{g\left(P_{0}^{\prime}-c^{2} \rho_{0}^{\prime}\right)}{\rho_{0} c^{2}}, \quad \text { and } \quad S_{\ell}^{2}=\frac{\ell(\ell+1) c^{2}}{r^{2}} .
$$

In these expressions ' denotes differentiation with respect to $r, P_{0}(r)$ is the background pressure, and $g$ is the local acceleration due to gravity. $\omega_{c}$ is an acoustic cutoff frequency which enters when the waves are not permitted to propagate beyond the solar surface. It therefore only plays a minor role deep inside the sun. Notice that $N^{2}<S_{\ell}^{2}+\omega_{c}^{2}$ is satisfied everywhere within the sun.

Once the radial displacement, $\xi_{r}$, is obtained by solving eq. (11), the radial part of the density perturbation, $\varrho(r)$, is found (except for very small $r$ ) using the following relation:

$$
\varrho(r)=\frac{1}{\left(S_{\ell}^{2}-\omega^{2}\right) r^{2}}\left[\omega^{2} \frac{d}{d r}-\frac{S_{\ell}^{2} N^{2} \rho_{0}}{d P_{0} / d r}\right]\left(r^{2} \rho_{0} \xi_{r}\right) .
$$

Eq. (11) is easily solved in the WKBJ approximation, with solutions

$$
\xi^{ \pm}(r)=K(r)^{-1 / 4} \exp \left[ \pm i \int^{r} d x \sqrt{K(x)}\right]
$$

In this approximation the solutions therefore propagate when $K>0$, and are damped when $K<0$. This implies the existence of two separate frequency intervals for propagating waves, depending on the size of $\omega$ in comparison with $N$ and $S_{\ell}$ :

$$
\begin{array}{cll}
\omega<|N| & : & g \text {-waves } \\
\omega>\sqrt{S_{\ell}^{2}+\omega_{c}^{2}} & : & p \text {-waves }
\end{array}
$$


The turning points for each kind of wave (which delimit the propagating regions) are defined as those radii for which $K$ changes sign, i.e. those $r$ for which $\omega^{2}=N^{2}(r)$ or $\omega^{2}=S_{\ell}^{2}(r)+\omega_{c}^{2}(r)$. Figure (3) plots the frequencies $N(r)$ and $S_{\ell}(r)$ as functions of radius within the sun, from which information the positions of the turning points for the various helioseismic waves may be inferred.

Because they are confined within the solar volume, helioseismic waves have quantized frequencies. Within the WKBJ approximation the allowed frequencies are determined by the condition:

$$
\int_{r_{1}}^{r_{2}} K(r)^{1 / 2} d r=\left(n-\frac{1}{2}\right) \pi ; \quad n=1,2, \ldots .
$$

Here $r_{1,2}$ denote the wave's turning points, and $n$ is its radial quantum number.

Typically, the larger the radial and angular quantum numbers, $n$ and $\ell$, are, the better the various approximations we have used - such as Cowling and WKBJ - become. We emphasize, however, that for the purposes of the present paper, all of the approximations used above are sufficiently accurate even for small $\ell$ and $n$.

We obtain numerical solutions of eq. (11) by matching linear combinations of the solutions, eq. (14), across the turning points. Figure (4) plots some sample $p$-wave profiles which were found in this way. Figure (5) gives similar plots for a few sample $g$-waves.

Several general properties of both $p$ - and $g$-waves emerge from an inspection of Fig. (3), and from the above discussion:

1) The square of the buoyancy frequency, $N^{2}$, becomes negative inside the convective zone. Indeed, the instability which this implies for the corresponding $g$-modes is precisely the instability towards convection which defines the convective zone. It is a general feature, then, that $g$-waves are damped inside the convective zone, and so are much harder to observe on the solar surface than are $p$-waves. This is the main reason why $p$-waves have been observed on the solar surface while $g$-waves have not.

2) The maximal value obtained by $N(r)$ is $N_{\max } / 2 \pi \sim 500 \mu \mathrm{Hz}$, which is the largest frequency possible for $g$-waves. Similarily, since $S_{\ell}^{2}+\omega_{c}^{2}>N^{2}, N_{\max }$ also gives a lower bound on possible $p$-wave frequencies.

3) Since $S_{\ell}$ increases with $\ell$ and decreases with $r$ only $p$-waves having small $\ell$ can penetrate very deeply into the solar interior.

4) Since $g$-waves also have frequencies which grow with $\ell$, those with large $\ell$ typically have frequencies which saturate the allowed maximal value, $\omega \sim N_{\max }$. As a result, we also expect these waves to have their largest amplitudes for radii in the interval $0.1<$ $r / R_{\odot}<0.3$. This makes these waves potentially interesting for neutrino propagation 
since this is precisely where MSW resonance typically occurs. Furthermore, these waves are also damped more strongly in the convective zone since $\varrho \sim r^{-(\ell+3 / 2)}$, and so they are even harder to detect using measurements at the solar surface.

\section{3) Amplitudes at Resonance and Relevance for Neutrino Propagation}

We are now in a position to answer the key question. How big can these waves be near the neutrino resonance point? The answer to this question dictates the size of the contribution of these waves in the detailed simulations of the next section. The answer to this question is necessarily different for $p$ - and $g$-waves, since experimental observations are available for $p$-waves, but not for $g$-waves. We therefore handle each of these waves separately in what follows.

- Pressure waves:

Pressure waves are undamped in the convective zone, and so propagate right up to the surface where they can be observed. A huge number of these modes have indeed been identified by a number of different experiments (see e.g. [15], as well as the references found in [14]). The measured surface velocities of individual modes are found to be in the range of $10 \mathrm{~cm} / \mathrm{sec}$.

We determine the amplitudes of these waves by requiring that their energy reproduces the observed energy distribution as a function of frequency [16] (see also [14]). This distribution is observed to peak at a value $10^{28}$ erg when $\nu \sim 3 \mathrm{mHz}$.

We plot the resulting amplitudes in Fig. (6). Here we display the maximal amplitude which a $p$-wave can obtain anywhere within the innermost 0.5 solar radii, given that it is normalized to reproduce the observed energy distribution. Each dot on this figure gives the frequency and the maximum amplitude of a wave having a specific value of $n$ and $\ell$. Lines connecting dots which differ only in $n$, but not in $\ell$, are drawn to help guide the eye.

As may be clearly seen from this figure, the maximum wave amplitudes are typically of order of $\delta \rho / \rho_{0} \lesssim 10^{-10}$, making these waves irrelevant for neutrino propagation.

\section{- Buoyancy waves:}

Since buoyancy waves have not been observed, a determination of their amplitude is necessarily more uncertain. In this section we estimate their potential amplitude in two steps. First we ask how big it is possible for them to be without their having been detected. Then we ask how big they might plausibly be expected to be on theoretical grounds.

For the purposes of determining how large a $g$-wave could escape detection, we demand that the radial velocity of oscillation at the surface of the sun produced by the wave be less 
than $1 \mathrm{~mm} / \mathrm{sec}$. This is a conservative limit on which waves can escape detection because velocities this small are unlikely to be detectable for the immediate forseeable future [15].

Figure (7) gives a representation of the amplitude which various $g$-waves would have if their surface radial velocity were $1 \mathrm{~mm} / \mathrm{sec}$. Every dot in this plot gives the frequency, $\nu_{n \ell}$, and the maximum relative amplitude, $\delta \rho / \rho_{0}$, which is obtained in the interval $r / R_{\odot} \in$ $[0,0.5]$, for a wave labelled by a pair of quantum numbers, $n$ and $\ell$. Lines have been drawn to guide the eye, connecting points which share a common value for $\ell$ ( $\equiv \mathrm{L}$ in the figure). Clearly the figure shows that $g$-waves can exist having any amplitude and still escape detection, although any points in the figure which appear much above the horizontal dashed line representing a $1 \%$ density fluctuation should be considered unreliable due to the breakdown of the linearized hydrodynamic equations we use.

Fig. (7) also shows that $g$-waves having large $\ell$ and small $n$ are the ones which can be largest deep in the solar interior without much disturbing the solar surface. Large $\ell$ is preferred because of two of the properties outlined in the previous sections: $(i) g$-modes having large $\ell$ tend to have their maximum amplitudes near $r / R_{\odot} \sim 0.3$, making them largest near resonance, and $(i i) g$-waves have a factor which falls off with radius like $r^{-\ell}$, which, for large $\ell$, tends to suppress their influence at the solar surface.

Large-amplitude $g$-waves could therefore easily escape current detection. How large might these waves reasonably be expected to be? An answer to this requires a theory of how these waves are excited and damped. Waves which have an appreciable overlap with the sun's convective zone can be both excited and damped — with an efficiency believed to be roughly independent of $n$ - due to their interactions with this zone's turbulent dynamics. $p$-waves, and $g$-waves which penetrate sufficiently far into the convective zone, can be excited in this way.

A different mechanism applies for those $g$-waves which don't extend far enough beyond the radiative zone (see [17] and references therein). These modes are believed to be stimulated in the energy-producing core, since there any local compression of the solar medium slightly increases the nuclear reaction rates, leading to higher temperatures and pressures and so to still more compression. In the absence of radiative damping of the resulting wave, this production mechanism would lead to a runaway instability. Radiative damping, however, has an efficiency which increases with $n^{2}$, and is therefore least effective for $g$-waves of small radial order.

Depending on whether excitation or damping is most effective - a subject of current controversy - $g$-waves having $n \leq 3$ have been argued to have much larger amplitudes than is expected for run-of-the-mill helioseismic waves. In a linearized analysis these modes grow exponentially, until they are so large that nonlinear effects start to saturate their growth 
[18]. An investigation of such nonperturbative effects argues that these low- $n$ modes could have energies as large as $10^{35-37} \mathrm{erg}[17][18]$ — some 10 orders of magnitude higher than the energies of other $g$ - and $p$-waves.

The modes for which this instability may apply are indicated in Figure (7) by the shaded area. In this shaded area, the thick, short straight line corresponds to a wave energy of $10^{36} \mathrm{erg}$. Any mode which lies below this line in the figure could not have an energy as large as $10^{36}$ ergs without also having a radial surface velocity larger than $1 \mathrm{~mm} / \mathrm{sec}$, increasing their chances of detection. Conversely, modes which lie above the thick black line have larger values for $\ell$, and so are damped more strongly with increasing $r$. They could therefore have energies as large as $10^{36} \mathrm{erg}$ and still have surface velocities which are too small to be detected.

Thus, large-amplitude $g$-waves might exist in the sun, although some caveats must apply. First, the runaway excitation of $n \leq 3 g$-modes is controversial [17]. Even should such a runaway excitation mechanism exist, it would have to preferentially excite modes having large $\ell$, since low $\ell$ modes are unlikely to have escaped detection if their energies become too large. In what follows we ignore such doubts, and restrict ourselves to computing the influence such waves would have on neutrino oscillations. We do so in order to see whether $g$-waves can affect the observed neutrino spectrum, even under the most extreme circumstances.

\section{Implications for Solar Neutrinos}

Given the helioseismic wave profiles and normalizations of the previous section, we are now positioned to compute their implications for neutrino evolution. We start with a brief description of some of the issues which arise with our numerical simulations, and then move on to the presentation of our results.

\section{1) The Numerical Algorithm}

Our numerical simulations were performed in the following steps.

1) We first generate the wave profile for the desired helioseismic wave.

2) We compute the survival probability for a single electron neutrino which propagates through this wave. In the interests of speed of calculation this was done simply by using Parke's formula (as derived by Parke - without fluctuations) for the survival probability. We verified for a sample of waves that this accurately reproduced a direct numerical solution of the MSW Schrödinger equation for the flavour degrees 
of freedom in the presence of the helioseismic wave. Agreement was good, although large-amplitude waves could cause neutrinos to pass through multiple resonances, and so it was necessary to use the Parke formula as applied to multiple resonance crossings in order to get agreement with numerical results.

3) Step (2) was repeated for an ensemble of 200 different density profiles, obtained by choosing the phase of the wave to be a random variable. The survival probability for each density profile was then averaged over the ensemble.

4) For comparison, the generalized Parke formula was also used to compute the ensembleaveraged survival probability.

We record here a number of detailed issues which better define our numerical procedure:

- Averaging Neutrino Production Sites: Although frequently ignored in theoretical calculations, neutrinos are not created exactly at the solar centre, and are instead produced throughout the solar core, out to several tenths of a solar radius. Furthermore, different nuclear reaction cycles produce neutrinos in different parts of the core, since their rates depend strongly on temperature. In our code we have used the production distributions of the Bahcall-Pinsoneault SSM [19] for the Beryllium, Boron and the proton-proton reactions.

The result of such an average for standard MSW oscillations without fluctuations, is shown in fig. (8). To generate this figure neutrinos are randomly produced (using Peter LePage's VEGAS Monte-Carlo routine) throughout the sun with probability given by the Bahcall-Pinsoneault distribution functions. For practical reasons, the simulations are done using around 200 neutrino creation sites. This is sufficient to obtain a precision of around $1 \%$ in the Monte-Carlo integration over the neutrino production volume. For simplicity we use spherical coordinates centred at the solar centre, with the positive $z$-axis pointing toward the earth.

- Neutrino Path Geometry: Since neutrinos are created at different points within the sun, they see different density profiles as they travel toward the earth depending on their initial creation point. In our calculations we evolve each neutrino along a path parallel to the $z$-axis, starting at its production point. This is the path the neutrino would take towards the earth, if the earth were infinitely far from the sun. It is a very good approximation to the real path taken to the earth because the maximum "inclination" of this path is of order $\theta \sim R_{\text {sun }} / R_{\text {sun-earth }} \sim 0.001$.

- Multiple Resonance Crossing: Since neutrinos are created throughout the solar centre, they may cross the resonance region more than once. This can happen if the neutrino 
starts further from the solar centre than is the resonance radius, but heads back through the solar centre on its way to the earth. Such neutrinos can cross the resonance density twice, in which case the jump probability in the Parke (or generalized Parke) formula should be replaced by $P_{J} \rightarrow 2 P_{J}\left(1-P_{J}\right)$.

Similarly, a neutrino passing through a large-amplitude helioseismic wave can pass through resonance more than twice. This can happen because the presence of the wave can make the solar density oscillate back and forth across the resonance density several times. A similar generalization of the jump probability is required in this case.

- More Than One Wave: A limitation of our simulations is our assumption that only one helioseismic wave is excited. In reality most modes are excited simultaneously, increasing the fluctuations seen by an ensemble of neutrinos. In principle, the effect of exciting many modes is computed by directly including all such modes into the numerical ensemble average. Our crude estimate of how our results would change is to use an 'equivalent amplitude' for a single wave. For instance, if $N$ waves were to contribute a roughly equal amount to the change in the neutrino survival probability, and suppose a $1 \%$ density fluctuation is required for any one of these waves to have an observable effect. Then we estimate that if all $N$ waves are present together, then the same observable effect would be produced if each wave only had an amplitude of $(1 / \sqrt{N}) \%$. This kind of scaling is justified within the master-equation approach for Gaussian fluctuations when each mode is uncorrelated with all of the others ${ }^{2}$.

\section{2) Numerical Results}

We now turn to a discussion of the results of these numerical simulations. Although we have run simulations of neutrino propagation through many helioseismic waves, we focus here on those $g$-waves having radial degree $n \leq 3$, since these are the ones that could have the largest amplitudes. Furthermore we consider only those modes whose radial surface velocities are smaller than $1 \mathrm{~mm} / \mathrm{sec}$, which corresponds to choosing $\ell \gtrsim 13$, (see Fig. (7)). These waves are superimposed on a background density profile, which we take to be the Bahcall-Pinsonneault SSM electron density profile [19].

Figures (9) and (10) give a typical result for the size of the change in the electronneutrino survival probability as a function of the maximum amplitude of the fluctuation. The dashed family of curves in these figures gives the generalized Parke prediction for an

2 Notice that a random superposition of the relevant g-waves will not significantly decrease the involved correlation length since they all peak in the same area within the sun and have similar wavelengths. 
$n=1, \ell=13$ (Fig. (9)) and an $n=3, \ell=50$ (Fig. (10)) $g$-wave having maximum amplitudes of $0.3 \%, 1 \%, 3 \%$ and $10 \%$ within the radial interval $r / R_{\odot} \in[0,0.5]$. These curves differ strongly from the MSW prediction throughout the resonance region, in agreement with the earlier, preliminary estimates of Refs. [4], [6], [7] and [8]. By contrast, the solid and dotted curves give the result of a direct numerical ensemble average, for the various amplitudes indicated, as described in the previous sections. These latter curves are indistinguishable from the MSW prediction for all values of $E / \Delta m^{2}$, even for fluctuations as large as $3 \%$ in size.

At first sight the small size of the predicted effect is surprising, given the fact that the $g$-wave of interest is strongest for $r / R_{\odot} \in[0.1-0.3]$, which is exactly where ${ }^{7}$ Be neutrinos pass through resonance. This small result is the consequence of the long-wavelength of the wave in question, which is of order $R_{\odot} / 10 \sim 7 \times 10^{4} \mathrm{~km}$. This long wavelength is much longer than the typical neutrino oscillation lengths, taking it well outside the domain of validity of the master-equation approach. This puts us in the large correlation length limit of Fig. (1), where deviation from the MSW effect becomes negligible.

Notice that the main difference between Figure (10) ( $n=3, \ell=50$ g-wave) as opposed to Figure (9) ( $n=1, \ell=13 \mathrm{~g}$-wave) is a decrease in size of the effect when computed using the generalized Parke formula and an increase in size when employing the direct numerical ensemble average, the latter leading to sizeable effects when the wave amplitude exceeds $3 \%$ (dotted curves in Fig. (10)). This difference is due to a decrease in correlation length when going from a mode of radial order $n=1$ to $n=3$. Two remarks are in order when comparing these figures. First, the difference in $\ell$ does is not responsible for the difference between these figures, since the neutrinos in these simulations were taken to originate in the solar centre. As a result, a larger value for $\ell$ doesn't much change the correlation length as seen by the outgoing neutrino. Second, the comparably large effect shown for the dotted $\left(\delta \rho / \rho_{0}=6 \%\right)$ curve in Figure (10) is an artifact of starting all neutrinos at the solar centre, since those produced further out miss the first upward 'bump' in the radial density profile, leaving the remaining downward and upward bumps to largely cancel. Once averaged over neutrino production sites, the effect on neutrino oscillations is drastically reduced, so much that evan a $g$-mode having $\delta \rho / \rho_{0}=1$ generates no detectable deviation from the MSW spectrum.

With this result it is clear that helioseismic waves can have no observable effect on neutrino oscillations. Even if a $g$-wave were imagined having a maximum amplitude as large as $1 \%, 3$ the suppression of its influence on neutrino oscillations due to its long

3 Notice that even though such modes don't produce detectable surface motion, and although their time-dependence prevents them being ruled out by seismic inversion methods, waves having this amplitude 
wavelength would even so cause it to negligibly alter neutrino oscillations. This disagrees with all previous predictions because these are based on the master-equation approach, which fails for waves having wavelengths as large as these.

\section{Conclusions}

We close with a summary of our conclusions, and a few speculations on how our results may affect other, more exotic, types of solar-neutrino solutions.

\section{1) Summary of Results}

In this paper we have carefully modelled neutrino passage through helioseismic waves, and have shown that helioseismic waves induce no observable consequence on MSW oscillations. Conversely, if MSW oscillations should be observed in the next generation of neutrino telescopes, then experimental agreement with MSW predictions provides no constraint on the size of density fluctuations in the resonance region that can be probed using linearized methods.

There are four main reasons underlying our conclusion. These are:

1) Neutrino oscillations appear to be sensitive to short-range fluctuations that are a few percent in amplitude in the immediate vicinity of any MSW resonance. With the possible exception of low- $n$, large- $\ell g$-modes, however, helioseismic waves are many orders of magnitude too small at resonance depths within the sun to affect neutrino oscillations.

2) Low- $n$, large- $\ell g$-waves can have much larger amplitudes because they are potentially subject to an instability to which other modes are insensitive. Unfortunately, such modes have wavelengths along the neutrino line of sight which are very large compared to neutrino oscillation lengths. The same is true of random combinations of many such modes, since all low- $n$, large- $\ell g$-modes tend to peak at the same depths within the sun, and to have similar wavelengths. This puts these waves beyond the domain of applicability of all previous calculations, and turns out to preclude even these potentially runaway modes from affecting neutrino oscillations.

3) By providing the first calculation which goes beyond the small-correlation-length approximation, we have found that long-wavelength waves produce effects which are additionally suppressed compared to previous estimates. We therefore find an additional

have enormously high energies of about $10^{46} \mathrm{erg}$ ! 
requirement if fluctuations are to significantly influence neutrino oscillations: the correlation length of the fluctuation should be roughly the same size as the perturbation length scale, which we define to be the length scale at whcih the master-equation approach fails. This length scale is typically of the same order of magnitude as the neutrino oscillation length.

4) Finally, any effect whose signature is a smearing of the adiabatic dip in the MSW survival probability profile, can potentially be masked by the similar smearing which happens purely within the ordinary MSW framework when the average over the neutrinoproduction site is taken (see fig. (8)).

We conclude that density fluctuations in the sun are unlikely to modify the small angle MSW neutrino spectrum. Most of the known fluctuations in the sun are too small at the neutrino resonance point to affect the predicted neutrino spectrum. The same is not true for helioseismic waves, but these nevertheless also neglibly influence neutrino oscillations. We know of no other plausible mechanism which can influence MSW oscillations.

\section{2) Implications for nonMSW Solutions to the Solar Neutrino Problem}

One of the major conlusions to emerge from the study of the influence of density perturbations on neutrino propagation is that the survival probability is sensitive almost exclusively to fluctuations at or close to the resonance [8]. MSW resonances are not influenced by most solar fluctuations simply because most fluctuations don't take place near the MSW resonance.

The present study adds to this the additional information that the correlation length should be comparable to the oscillation length, if a fluctuation is to appreciably influence neutrino oscillations.

Although MSW oscillations always occur well away from the convective zone, and so well away from its associated density fluctuations, this is not true for some other proposals to solve the solar neutrino problem. In particular, it is not true for magnetic-moment solutions, in which neutrinos are conjectured to posses a small magnetic moment which induces flavour oscillations in the presence of large magnetic fields [20]. In this scenario neutrinos can also undergo resonant conversions in the presence of matter, with the important difference that some of these resonances can occur within the convective zone. The survival probability could therefore in principle be much more strongly modified by the strong fluctuations in the magnetic field and electron density within the convective zone. The conclusions of this paper show that in order to produce an effect any such fluctuations must also have correlation lengths which are comparable to the typical oscillation 
lengths for these neutrinos. The potential interplay between convective-zone fluctuations and resonant magnetic-moment oscillations would bear closer study.

\section{Acknowledgements}

We would like to acknowledge helpful correspondence with Professors Joergen ChristensenDalsgaard, Pawan Kumar and John Bahcall concerning helioseismology. Our research is financially supported by NSERC of Canada and FCAR du Québec. 


\section{References}

[1] R. Davis, D.S. Harmer and K.C. Hoffman, Phys. Rev. Lett. 20 (1968) 1205;

J.K. Rowley et.al., in Solar Neutrinos and Neutrino Astronomy, AIP Conference Proceedings number 126, edited by M.L. Cherry, W.A. Fowler and K. Lande, (1985);

K.S. Hirata et.al., Phys. Rev. Lett. 65 (1990) 1297;

P. Anselmann et.al., Phys. Lett. 327B (1994) 377;

J.N. Abdurashitov et.al., Phys. Lett. 328B (1994) 234.

[2] The following references provide excellent reviews:

J.N. Bahcall, Neutrino Astrophysics, Cambridge University Press, 1989;

S. Turck-Chièze et.al., Phys. Rep. 230 (1993) 57;

W. Haxton, Ann. Rev. of Astr. and Astrophys. 33 (1995) 459.

[3] L. Wolfenstein, Phys. Rev. D17 (1978) 2369;

V. Barger, K. Whisnant, S. Pakvasa and R.J.N. Phillips, Phys. Rev. D22 (1980) 2718;

P. Langacker, J.P. Leville and J. Sheiman, Phys. Rev. D27 (1983) 1228;

S.P. Mikheyev and A. Yu. Smirnov, Sov. Phys. Usp. 29 (1986) 1155; Yad. Fiz. 42 (1985) 1441 [Sov. J. Nucl. Phys. 42 (1985) 913]; Nuovo Cimento 9 (1986) 17;

S.P. Rosen and J.M. Gelb, Phys. Rev. D34 (1986) 969;

H. Bethe, Phys. Rev. Lett. 56 (1986) 1305;

W. Haxton, Phys. Rev. Lett. 57 (1986) 1271;

A.J. Baltz and J. Weneser, Phys. Rev. D37 (1988) 3364;

P.D. Mannheim, Phys. Rev. D37 (1988) 1935.

[4] F.N. Loreti and A.B. Balantekin, Phys. Rev. D50 (1994) 4762 (nucl-th/9406003);

F.N. Loreti, Y.Z. Qian, G.M. Fuller and A.B. Balantekin, Phys. Rev. D52 (1995) 6664 (astro-ph/9508106);

E. Torrente Lujan, preprint BUTP-96-8 (hep-ph/9602398).

[5] D. Michaud, McGill University M.Sc. thesis, 1994;

in the 17th proceedings of the Annual MRST Meeting, 1995. 
[6] H. Nunokawa, A. Rossi, V.B. Semikoz and J.W.F. Valle, Nucl. Phys. B472 (1996) 495 (hep-ph/9602307).

[7] A.B. Balantekin, J.M. Fetter and F.N. Loreti, Phys. Rev. D54 (1996) 3941 (astroph/9604061).

[8] C. P. Burgess and D. Michaud, Ann. Phys. (NY) 256 (1997) 1, (hep-ph/9606295); and contribution to the proceedings of the 17th International Conference on Neutrino Physics and Astrophysics (NEUTRINO '96), ed. by K. Enqvist, K. Huitu and J. Maalampi, Helsinki, World Scientific, (hep-ph/9611368), 1996.

[9] See, e.g., H.C. Spruit, Mem. S.A. It. (1996), astro-ph/9605020.

[10] J.N. Bahcall, M.H. Pinsonneault, S. Basu and J. Christensen-Dalsgaard, Phys. Rev. Lett. 78 (1997) 171.

[11] A very readable review is: Jorgen Christensen-Dalsgaard, Lecture Notes, available at http://bigcat.obs.aau.dk/ jcd/oscilnotes/.

[12] P. Krastev and A.Yu. Smirnov, Phys. Lett. 226B (1989) 341;

W. Haxton and W.-M. Zhang, Phys. Rev. D43 (1991) 2484.

[13] S.J. Parke, Phys. Rev. Lett. 57 (1986) 1275.

[14] S. Turck-Chièze et.al., Phys. Rep. 230 (1993) 57.

[15] B. Fleck, Rev. Mod. Astron. 10 (1997) in press, http://esa.nascom.nasa.gov / bfleck/Preprints/.

[16] K.G. Libbrecht, M.F. Woodard and J.M. Kaufman, Astrophys. J. Suppl. 74, (1990) 1129 .

[17] P. Kumar, E.J. Quataert and J.N. Bahcall, Astrophysical Journal 458, (1996), L83.

[18] P. Kumar and J. Goodman, Ap. J. in press, (1996).

[19] J.N. Bahcall and M.H. Pinsonneault, Rev. Mod. Phys. 67 (1995) 781.

[20] A. Cisneros, Astro. \& Space Sci. 10 (1971) 87; 
M.B. Voloshin, M.I. Vysotskii and L.B. Okun, Sov. Phys. JETP 64 (1986) 446;

C.S. Lim and W.J. Marciano, Phys. Rev. D37 (1988) 1368;

E.K. Akhmedov, Phys. Lett. 213B (1988) 64. 


\section{Figure Captions}

(1) Here we compare the two averaging procedures described in the text in the context of the 'Cell' model. We show the survival probability as a function of cell size (i.e. correlation length), $\ell$, using the following set of neutrino parameters: $\delta m^{2} / 2 k \sim$ $10^{-6} \mathrm{eV}^{2} / \mathrm{MeV}, \sin ^{2} 2 \theta_{V}=0.01$ and $\epsilon=0.1$. $\left\langle n_{e}\right\rangle$ was chosen to fall exponentially along the neutrino-propagation $(z)$ direction, with scale height $h=R_{\odot} / 10 \sim 6.6 \times 10^{4}$ $\mathrm{km}$. The horizontal dotted line gives the MSW result, while the result obtained using the generalized Parke formula is given as a thin solid line. The other three curves give the result obtained using the ordinary Parke formula for each neutrino, with the result averaged over a random ensemble of 200 density profiles of the Cell type. The thick solid line adjusts the cells to ensure that the MSW resonance always occurs inside a cell. The dashed curve similarly adjusts the cells to ensure that the resonance occurs precisely at a cell boundary. The dot-dashed curve gives the result for randomly distributed cell positions. The differences between these curves show the sensitivity of the result to the details of how the cells are defined, and in particular to the sharp density gradient we have assumed between cells. The generalized Parke formula differs significantly from the others for cell sizes larger than about $10^{4} \mathrm{~km}$. Above this point the generalized Parke value for the survival probability remains fixed around $P_{e}=0.5$, whereas the 'real', numerically obtained, value decreases sharply to the MSW result. Notice also that a large density gradient right at the resonance, given here by a sharp cell boundary, can have a significant effect even for very large correlation length.

(2) This figure indicates the position of the MSW resonance within the sun as a function of neutrino energy and MSW parameters. The two solid lines delimit the range permitted for the small-angle MSW solution, whereas the dashed and dotted lines represent various options within the large-angle MSW solution. The main production regions for $p-p,{ }^{7} \mathrm{Be}$, and ${ }^{8} \mathrm{~B}$ neutrinos are indicated for comparison in the same figure as shaded areas.

(3) The solid line gives the buoyancy frequency, $N(r) / 2 \pi$, as a function of depth within the sun. The dashed lines similarly plot the acoustic frequency, $S_{\ell}(r) / 2 \pi$, for different values of $\ell(\equiv \mathrm{L})$. The two horizontal, dot-dashed lines represent the radial interval over which representative $p$ - or $g$-waves would be undamped. The $p$-wave indicated was assumed to have angular degree $\ell=10$.

(4) This figure plots the amplitude of a few sample $p$-waves against radius in the sun. The vertical axis gives the scaled quantity $\xi_{r}(r) r \rho_{0}^{1 / 2}(r)$ in arbitrary units, where $\xi_{r}(r)$ is 
the wave's radial displacement and $\rho_{0}(r)$ is the background mass density.

(5) This figure plots the amplitude of a few sample $g$-waves against radius in the sun. The vertical axis gives the scaled quantity $\xi_{r}(r) r \rho_{0}^{1 / 2}(r)$ in arbitrary units, where $\xi_{r}(r)$ is the wave's radial displacement and $\rho_{0}(r)$ is the background mass density.

(6) Here we plot the maximum amplitude which is attained by a pressure wave for $r<$ $0.5 R_{\odot}$. The waves are normalized by requiring them to reproduce the experimentallymeasured energy distribution as a function of frequency (see e.g. [14]). The interval chosen for $r$ is sufficiently generous to contain the MSW resonance for virtually all choices for neutrino mixing parameters. The points displayed give $\delta \rho / \rho$ as a function of linear frequency, $\nu_{n \ell}=\omega_{n \ell} / 2 \pi$, for $p$-waves having various angular $(\ell \equiv \mathrm{L})$ and radial $(n)$ quantum numbers. To guide the eye, solid lines connect points representing waves having a fixed value of $\ell(\equiv \mathrm{L})$, but varying $n$.

(7) Here we plot, as a function of linear frequency $\left(\nu_{n \ell}=\omega_{n \ell} / 2 \pi\right)$, the maximum relative amplitude, $\delta \rho / \rho$, which can be acheived by a $g$-wave for any $r$ less than half a solar radius. The dots are obtained by requiring that the radial velocity of the wave equal $1 \mathrm{~mm} / \mathrm{sec}$ at the solar surface. Each dot represents a wave having a different value of $n$ and $\ell(\equiv \mathrm{L})$. Thin solid lines are drawn to guide the eye which connect points having the same values of $\ell$, but differing values for $n$. The horizontal dashed line corresponds to a density wave whose maximum amplitude is $1 \%$ of the unperturbed mass density. An analysis of waves using linearized equations breaks down for points much above this line. The shaded area indicates which modes may be subject to instability [17], and so have much larger amplitudes. The thick short straight line corresponds to a wave energy of $10^{36} \mathrm{erg}$, which has been argued [17] to be an energy which these waves could easily aquire, provided they really are linearily instable [17]. Modes below this line would, if they had this energy, exhibit surface velocities above 1 $\mathrm{mm} / \mathrm{sec}$, increasing their chances of detection. We are mainly interested in overstable modes above the line, since these could have acceptably large energies while still having surface velocities which are small enough to escape detection.

(8) We plot here the MSW survival probabilities averaged over neutrino production points in the sun. The dotted, dash-dotted and dashed lines show the survival probabilities when averaged over the production points of $\mathrm{pp},{ }^{7} \mathrm{Be}$ and ${ }^{8} \mathrm{~B}$ neutrinos, respectively. This is to be contrasted with the solid line, which gives the result if all neutrinos are assumed to have been produced at the exact center of the sun. The average over the neutrino-production site is an important background to any fluctuation calculation, 
because both tend to smear out the MSW survival-probability curve.

(9) We plot here the survival probability for electron neutrinos propagating through the $n=1, \ell=13 \mathrm{~g}$-wave mode. Here the various dashed lines represent the predictions of the generalized Parke formula for varying amplitude fluctuations. The long-dashed line corresponds to: $\left(\delta \rho / \rho_{0}\right)_{\max }=0.3 \%$; the short-dashed line: $1 \%$ and the dotdashed line: $3 \%$. For comparison, the solid and dotted curves represent a direct Monte Carlo averaging over 200 density profiles, as described in the text. These are largely indistinguishable from the pure MSW prediction, even for fluctuations as large in amplitude as $\left(\delta \rho / \rho_{0}\right)_{\max }=1$ (dotted line). For simplicity of comparison this plot is performed assuming all neutrinos are produced right at the solar centre.

(10) Same as Fig. (9) the only difference being that here we let the neutrinos propagate through an $n=3, \ell=50 \mathrm{~g}$-wave mode. Again the various dashed lines represent the predictions of the generalized Parke formula. The long-dashed line corresponds to: $\left(\delta \rho / \rho_{0}\right)_{\max }=0.3 \%$; the short-dashed line: $1 \%$ and the dot-dashed lines: $3 \%$ and $10 \%$ respectively. The solid (1\% or less) and dotted (3\% and $6 \%)$ curves, on the other hand, represent a direct Monte Carlo averaging, as described in the text. These are largely indistinguishable from the pure MSW prediction, even for fluctuations as large in amplitude as 3\% (lower dotted line). For simplicity of comparison this plot is performed assuming all neutrinos are produced right at the solar centre. 


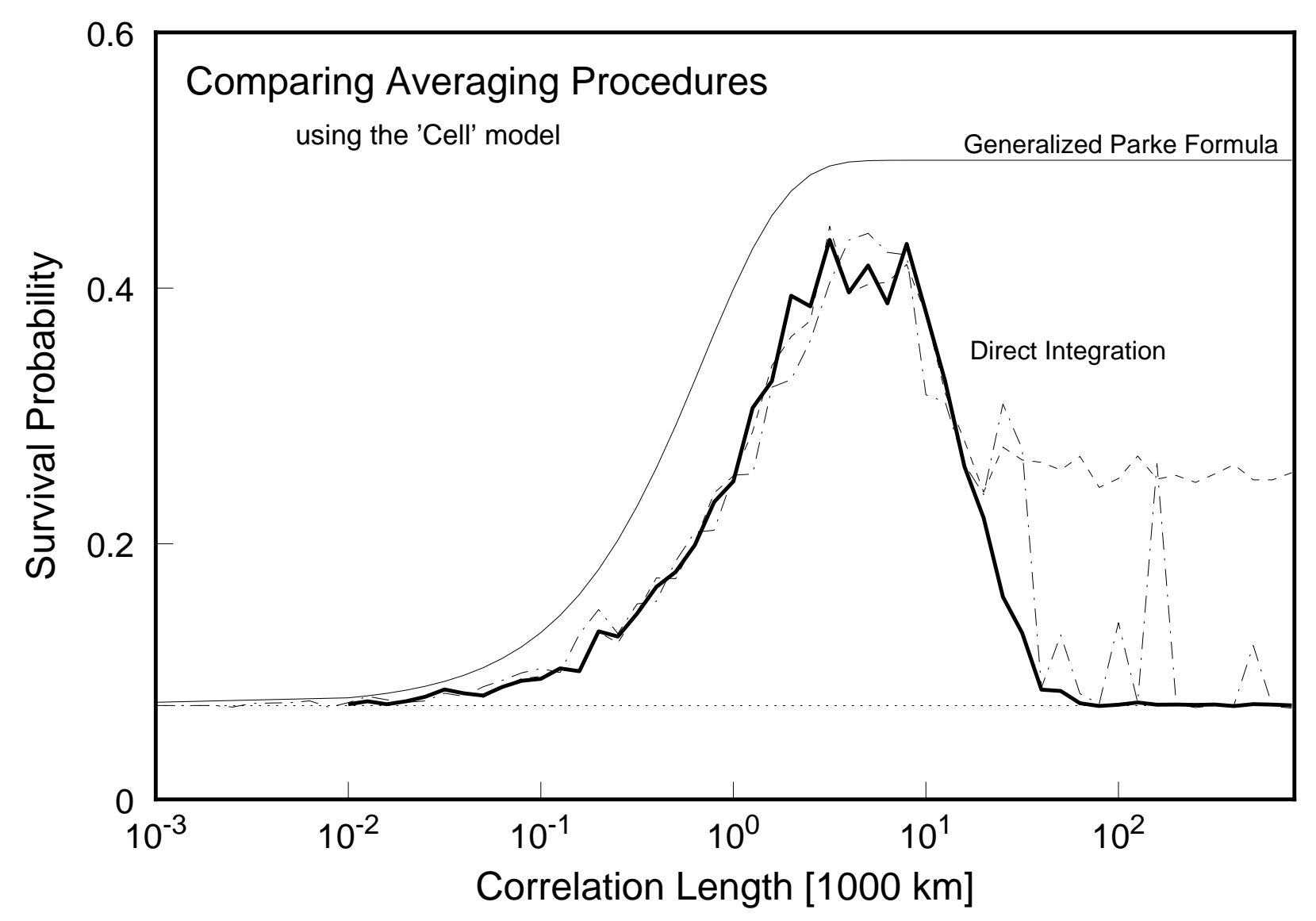




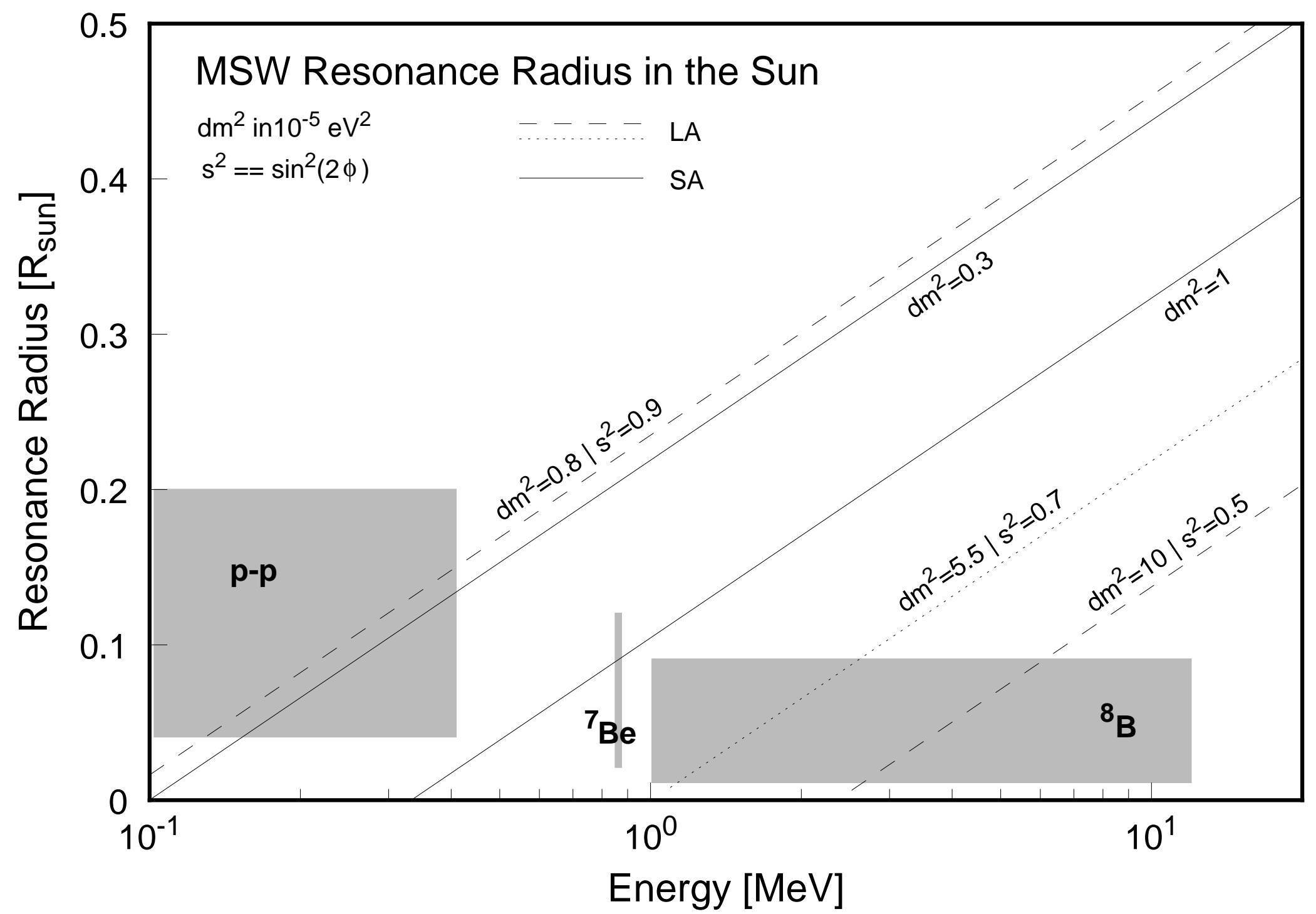




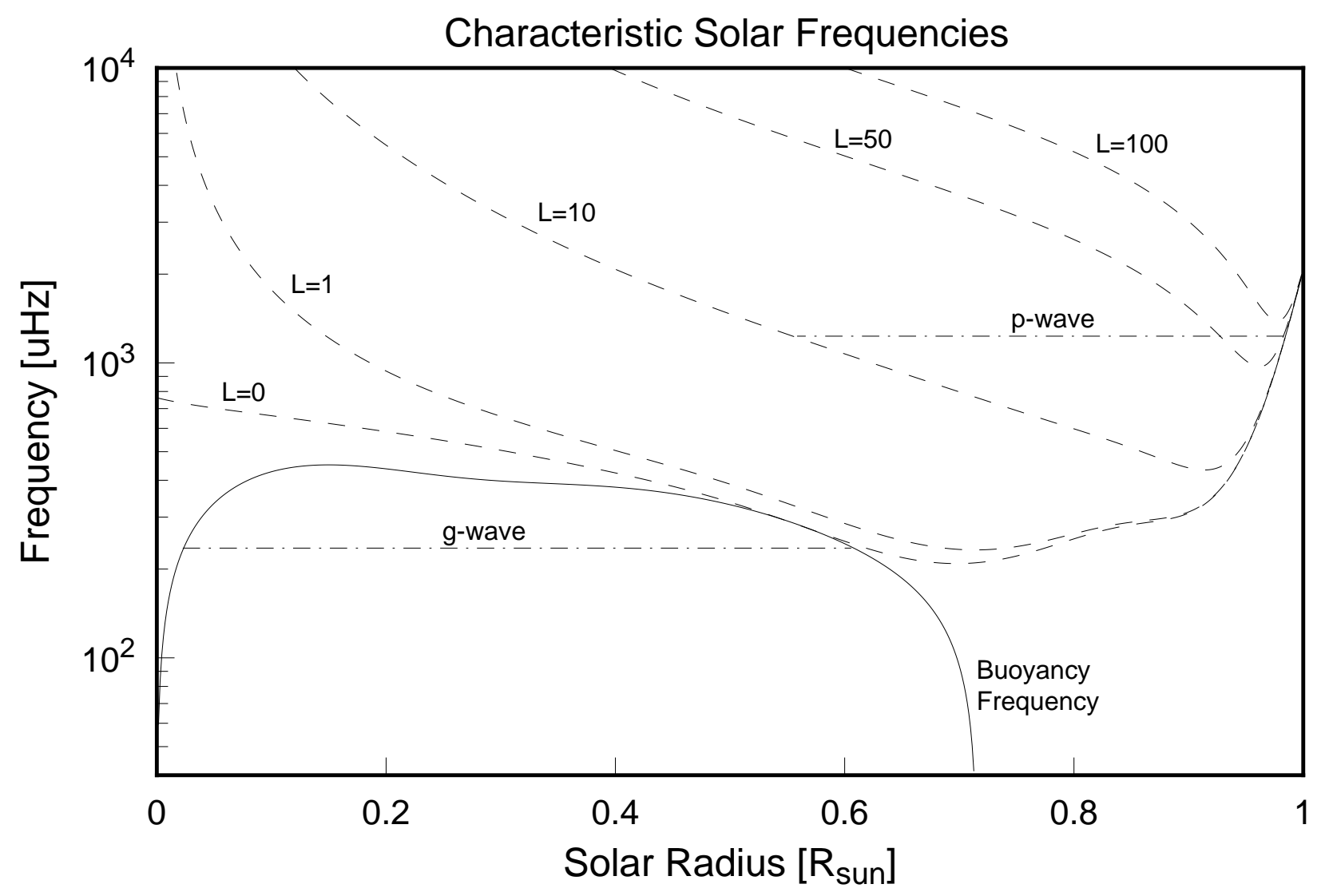




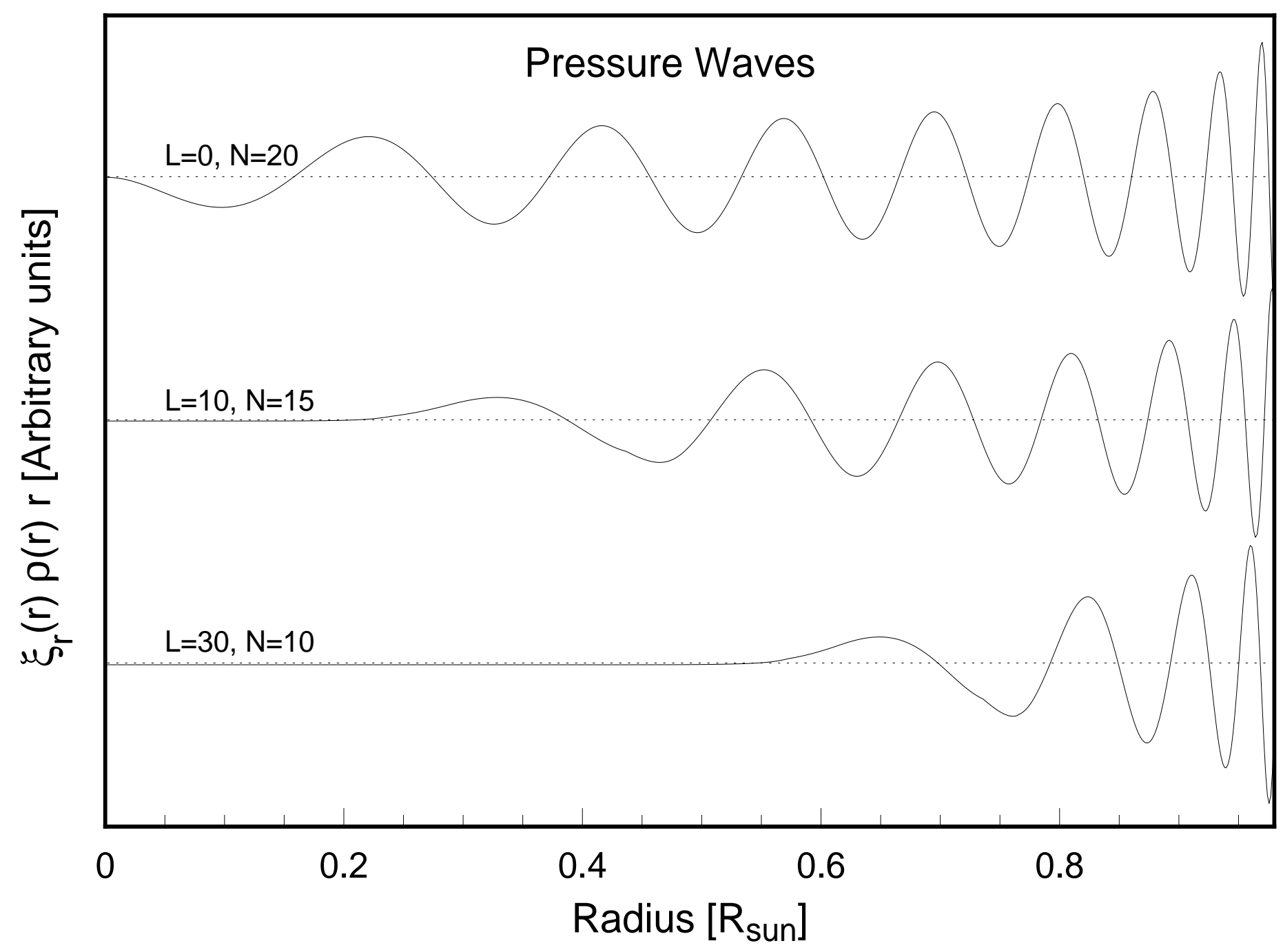




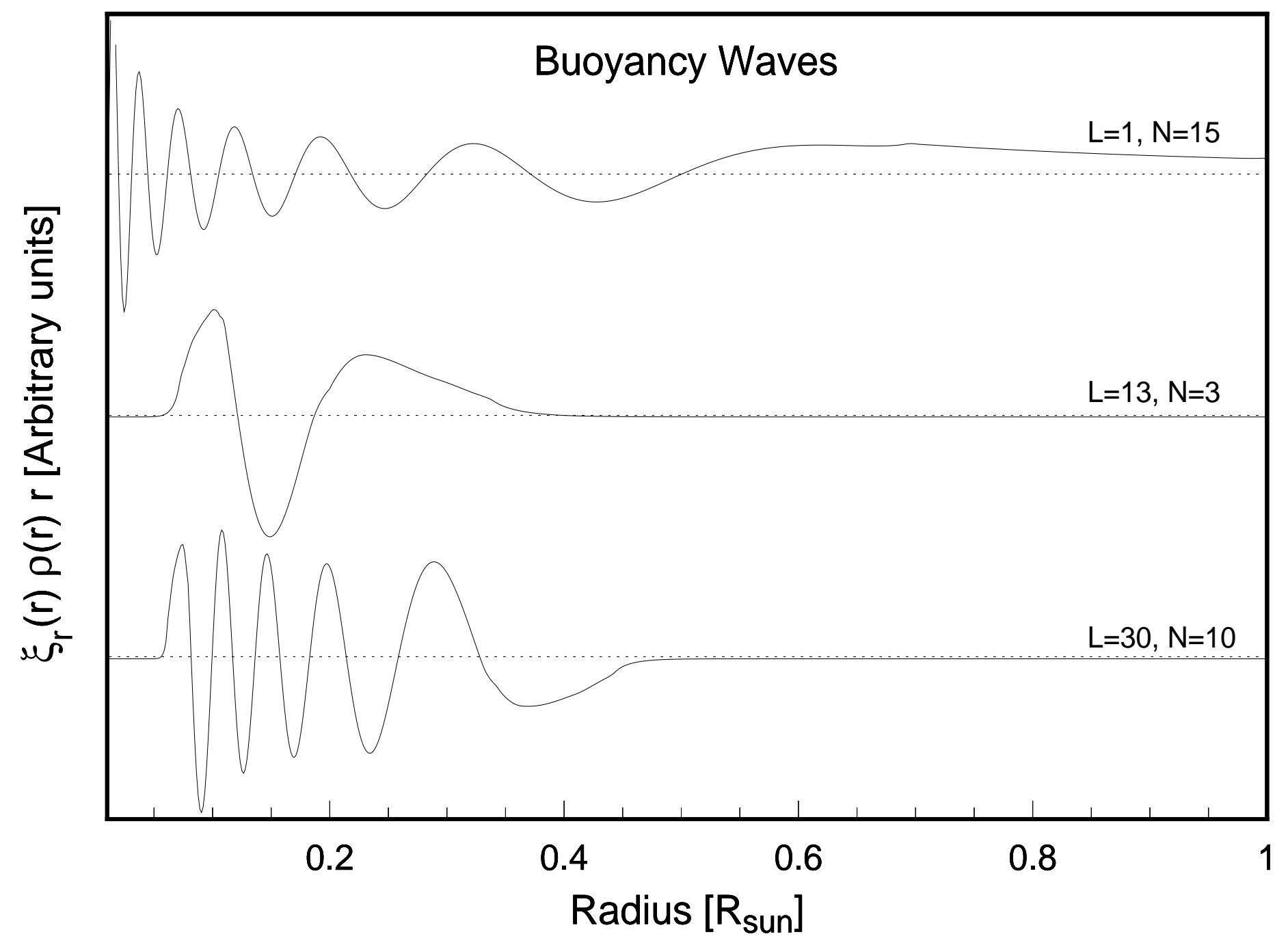




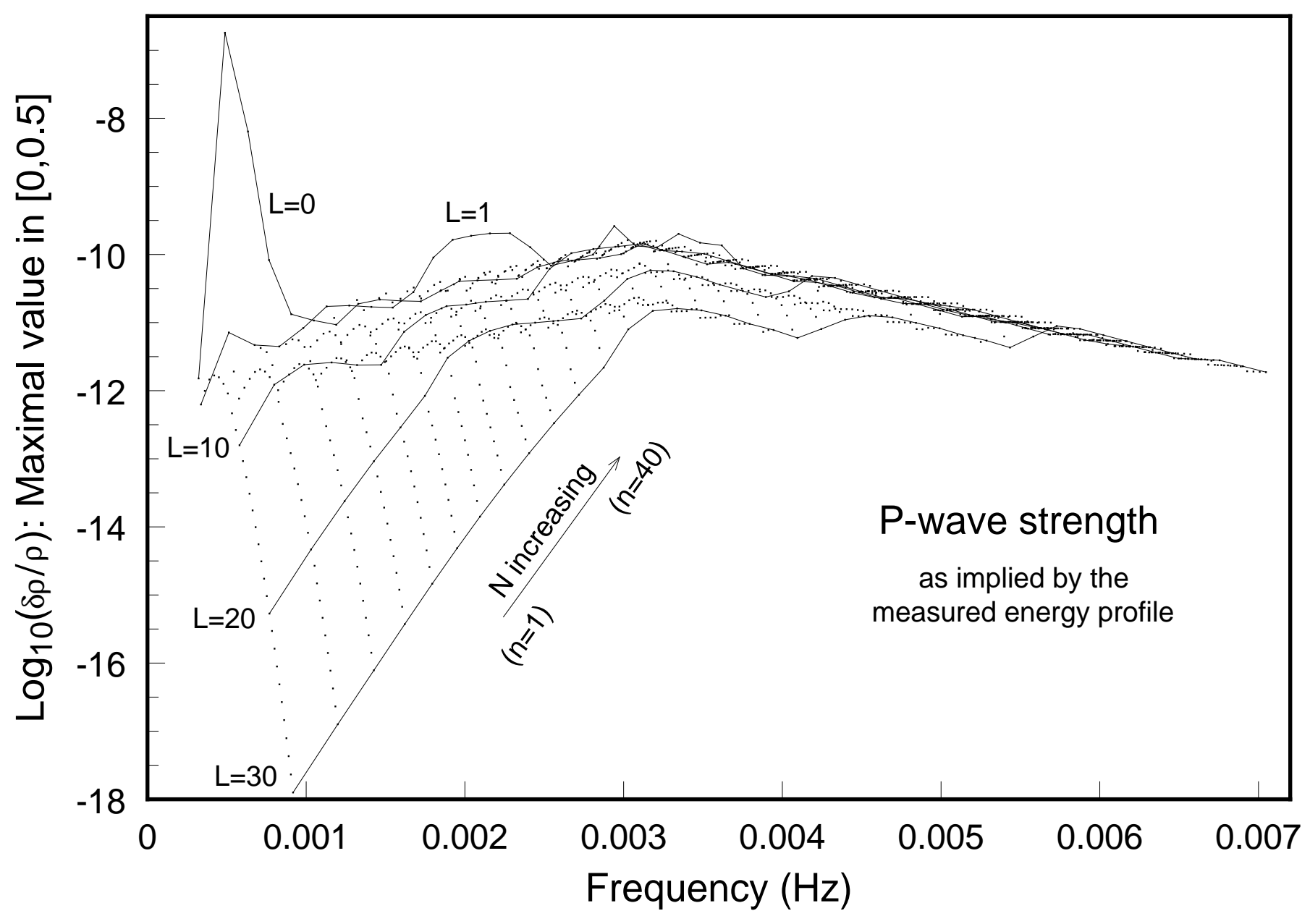




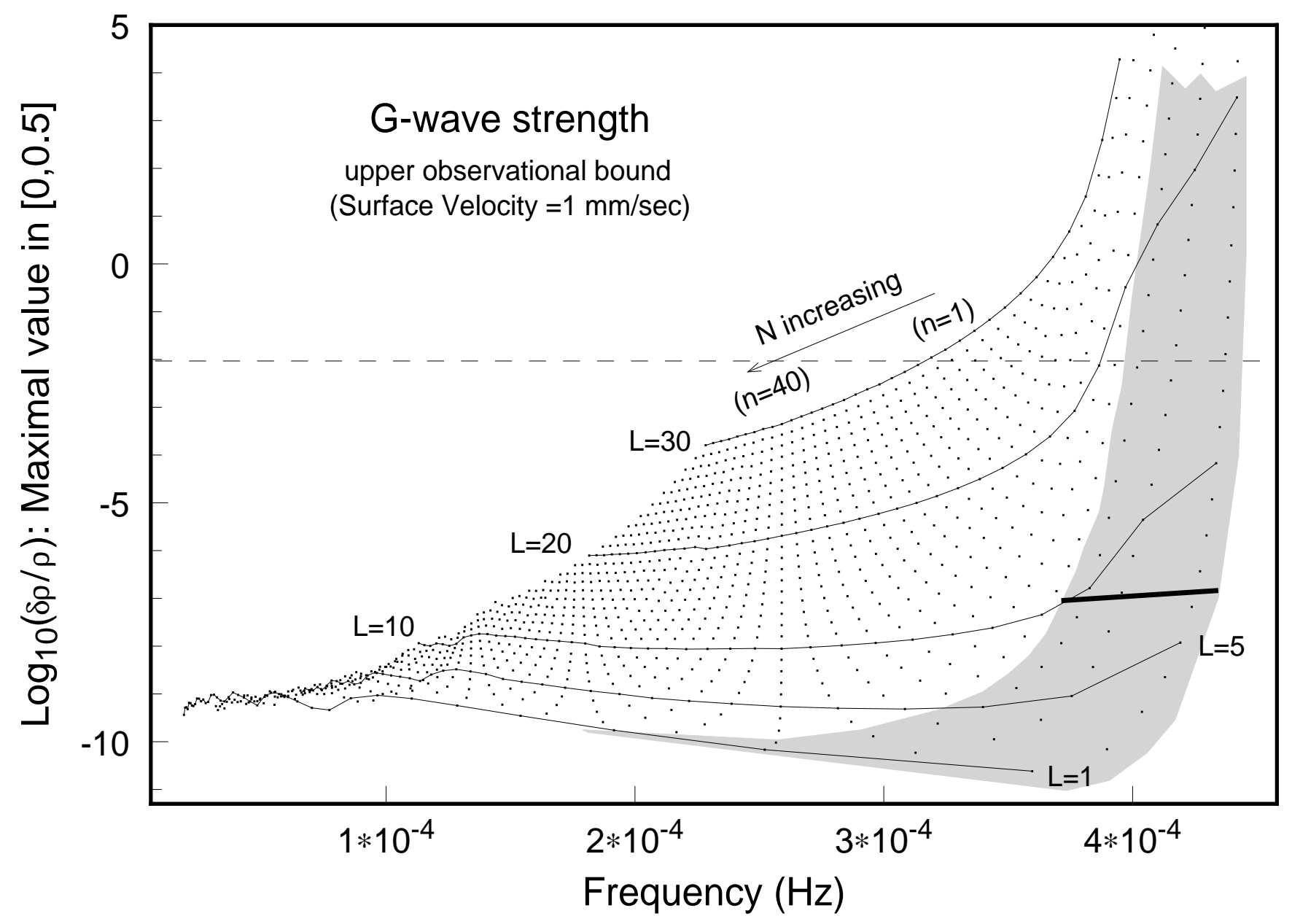




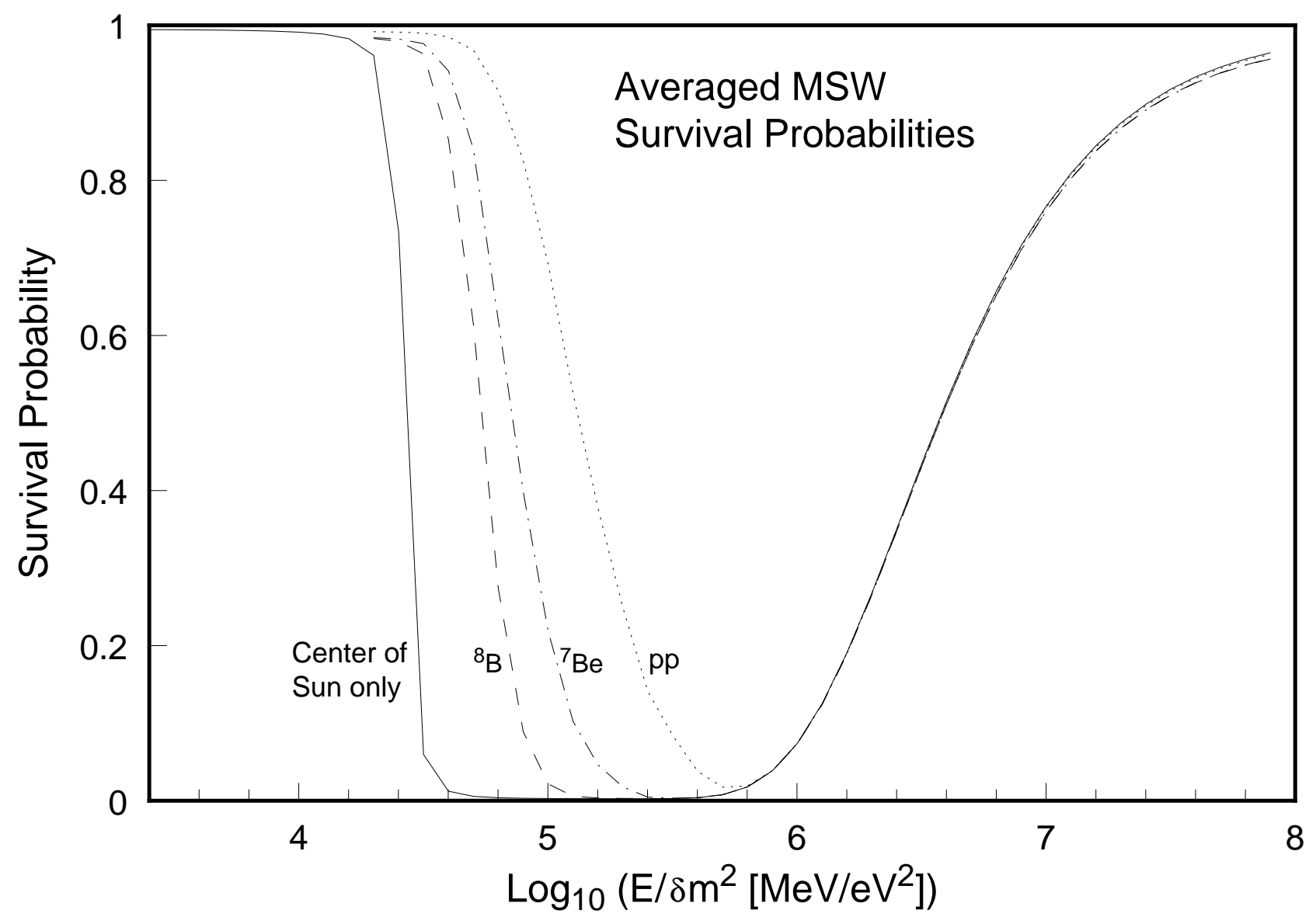




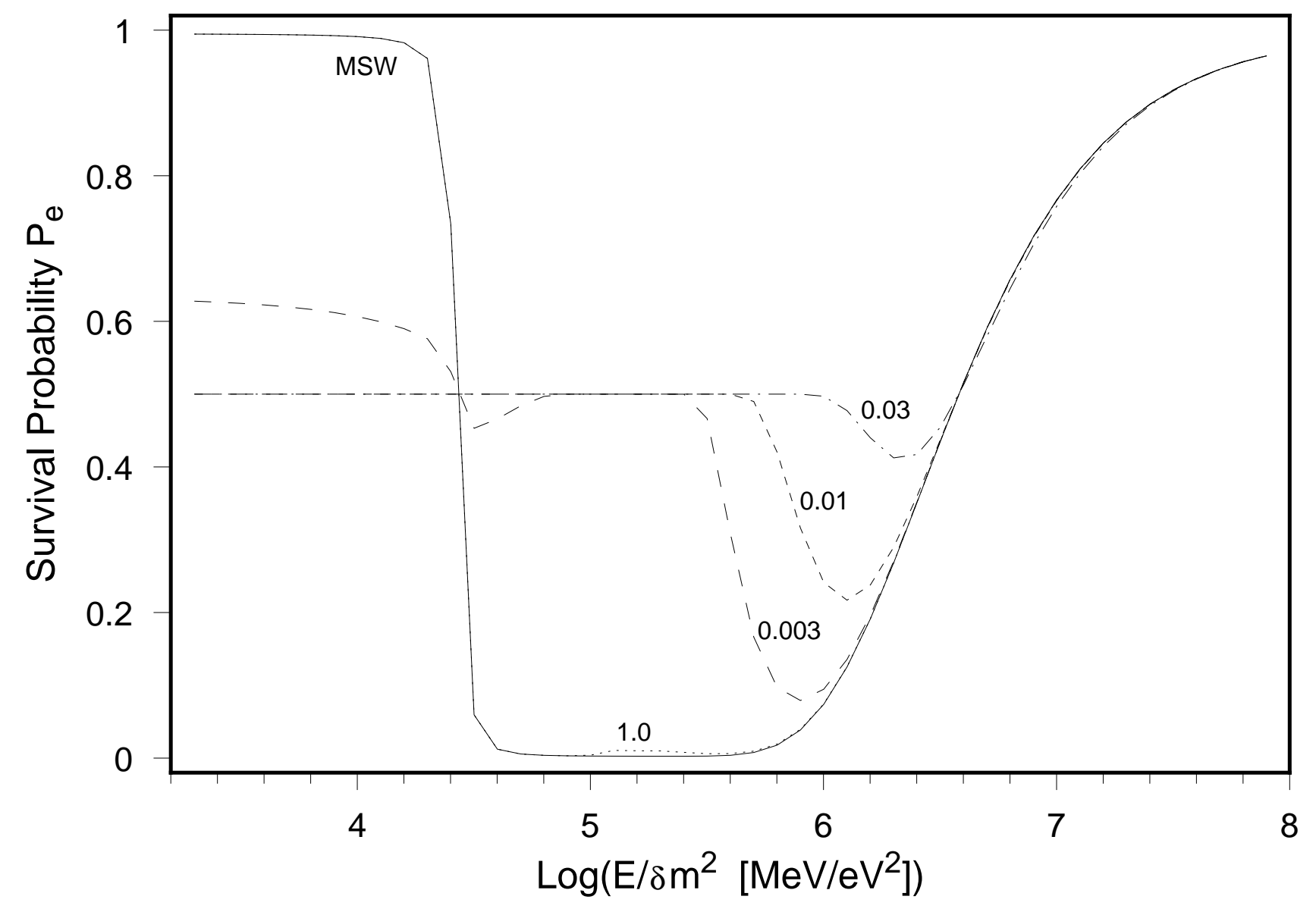




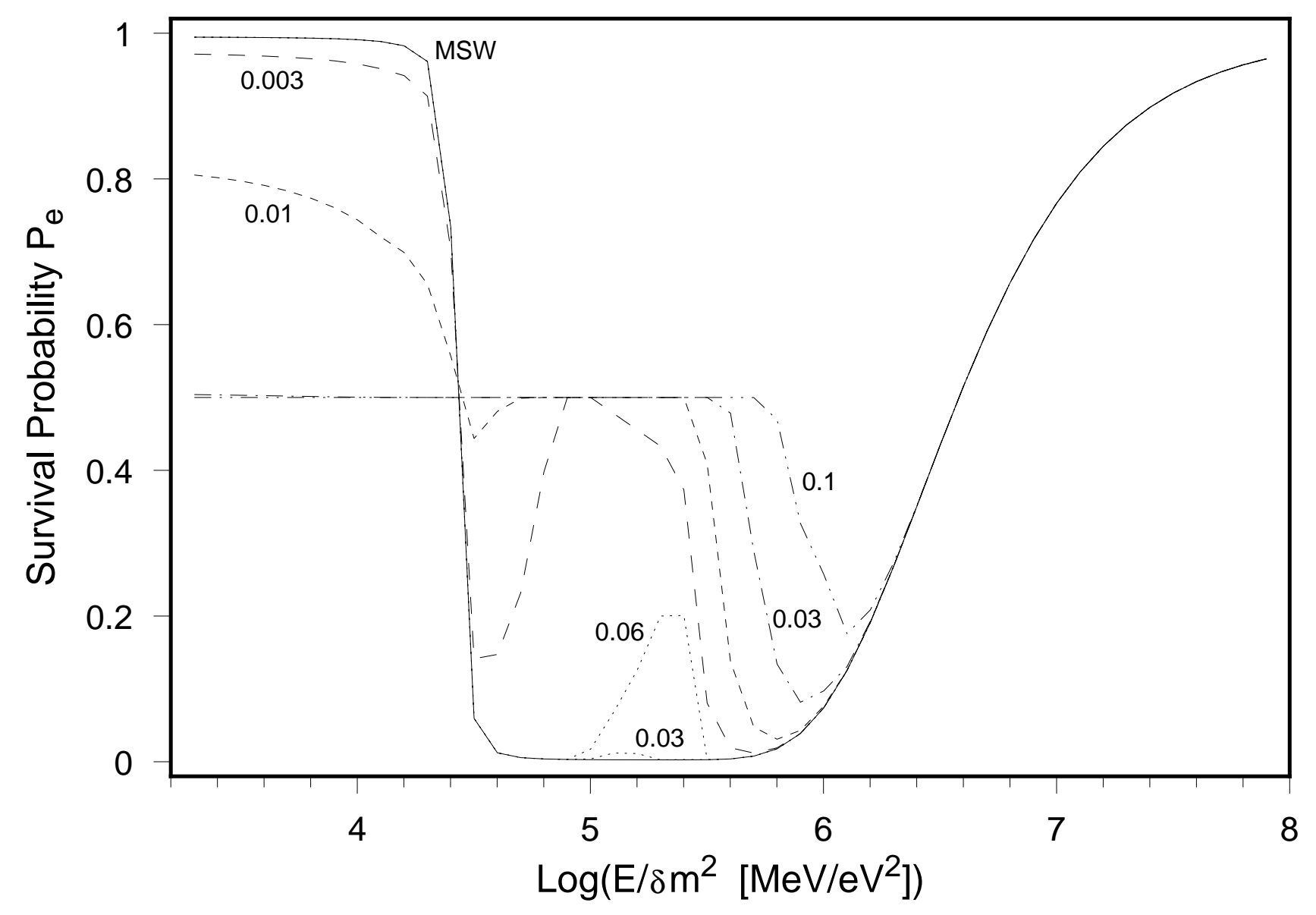

